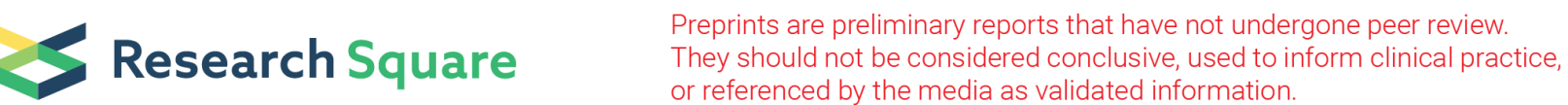

\section{Risk factor for postoperative hemorrhage after cesarean section for placenta previa : A retrospective study}

Hiroki Ishibashi

National Defence Medical College

Morikazu Miyamoto ( $\nabla$ morikazu1118@hotmail.co.jp)

National Defence Medical College https://orcid.org/0000-0003-4763-0926

Hiroaki Soyama

National Defence Medical College

Hideki Iwahashi

National Defence Medical College

Haruka Kawauchi

National Defence Medical College

Kazuki Takasaki

National Defenca Medical College

Hiroko Matuura

National Defence Medical College

Masaya Nakatsuka

National Defence Medical College

Taira Hada

National Defence Medical College

Masashi Takano

National Defence Medical College

\section{Research article}

Keywords: Cesarean Section, Intraoperative Hemorrhage, Placenta Previa, Postoperative Hemorrhage

Posted Date: September 30th, 2019

DOI: https://doi.org/10.21203/rs.2.13061/v2

License: (c) (i) This work is licensed under a Creative Commons Attribution 4.0 International License.

Read Full License 


\section{Abstract}

Background Placenta previa can cause postoperative hemorrhage. Even cases with less intraoperative hemorrhages during cesarean section have the potential risk of developing postoperative hemorrhage. However, there are few reports on the predictive factors of postoperative hemorrhage associated with placenta previa. The aim of this study was to identify the predictive factor for postoperative hemorrhage after cesarean section in women with placenta previa. Methods We identified women with placenta previa who underwent cesarean section at our institution between January 2003 and February 2015. All women who received any hemostatic procedure, such as intrauterine balloon tamponade and gauze infiltration during cesarean section were excluded. All women were classified into two groups: Group A, women with massive postoperative hemorrhage, defined as over $500 \mathrm{ml}$ of hemorrhage after cesarean section, and Group B, women without postoperative hemorrhage. A retrospective analysis to identify the predictive factors for postoperative hemorrhage was conducted. Results Out of 128 women, 10 (7.8\%) were included in Group A and 118 (92.2\%) in Group B. There was no statistically significant difference in maternal history between the groups. The number of women suspected to have placental adhesion was higher in Group A than in Group B $(p=0.006)$. Furthermore, the amount of intraoperative hemorrhage in Group A was higher than that in Group B $(p=0.025)$. As treatment for postoperative hemorrhage, more women in Group A received allogenic blood transfusion $(p=0.003)$ and uterine artery embolization $(p=$ $0.010)$. In univariate analysis, placental adhesion suspected by the surgeon during cesarean section was the predictive factor for postoperative hemorrhage with placenta previa $(p=0.002)$. Conclusions When placental adhesion is suspected by surgeons during a cesarean section, additional hemostatic procedures should be performed to prevent possible postoperative hemorrhage.

\section{Background}

Placenta previa can cause maternal and neonatal mortality and morbidity by massive hemorrhage $[1,2]$. Hence, more precise and appropriate strategies of prediction and preparation should be developed with the purpose of avoiding these adverse obstetrical outcomes [3,4]. Massive placenta previa hemorrhages occurs either in the operative or in the postoperative period [5]. Consequently, strategies for prediction and prevention of intra- and postoperative hemorrhages are required.

Previous reports have characterized several factors predicting massive hemorrhages in placenta previa, such as high maternal age, previous history of a cesarean section, placental adhesion, antenatal bleeding, and heavy birth weight. [6-9]. Also, imaging modalities are useful to predict intraoperative hemorrhage. The ultrasonographic (US) findings including sponge like consistency, complete placenta previa, anterior placentation, and shortening of a third-trimester cervical length $[6,7,9-15]$ and magnetic resonance imaging (MRI) findings such as uterine bulging, heterogeneous placenta, adjacent organ invasion, and cervical varicosities could predict placenta accrete [16-18]. Furthermore, effective strategies to decrease intraoperative hemorrhage in placenta previa include intrauterine balloon tamponade and perioperative temporary balloon occlusion of internal iliac artery $[19,20]$. 
Placenta previa is well known to induced massive postoperative hemorrhage [21]. Even cases with less intraoperative hemorrhage during cesarean section had the potential risk to develop massive postoperative hemorrhage [22]. Without rapid and appropriate treatment, massive postoperative hemorrhage might induce adverse obstetrical outcomes including maternal death [22]. As a strategy for massive postoperative hemorrhage associated with placenta previa, previous studies demonstrated that intrauterine balloon tamponade was a useful technique to reduce the hemorrhage [23-25]. In these reports, the balloon was inserted after the massive postoperative hemorrhage had occurred [23,24]. Once a massive postoperative hemorrhage develops, the mother requires several treatments including blood transfusion [21]. Therefore, if predictive factors of massive postoperative hemorrhage related with placenta previa are identified, hazardous situations can be avoided before massive postoperative hemorrhage develops. The purpose of this study was to identify predictive factors of massive postoperative hemorrhage after cesarean section in cases with placenta previa.

\section{Methods}

All singleton pregnancy cases that underwent cesarean section due to placenta previa at our institution, between January 2003 and February 2015, were identified. The inclusion and exclusion criteria were decided according to surgical procedures. The basic surgical procedure for placenta previa was performed as previously described by Soyama et al. [25]. Briefly, after abdominal wall incision, a transverse incision into the lower segment of the uterus was performed. In cases with a placenta in the anterior uterine wall, surgeons avoided the placenta and the incision was guided by ultrasound [25]. After delivery and until $24 \mathrm{~h}$ after the cesarean section, oxytocin 5 IU was started intravenously in a $500-\mathrm{mL}$ saline drip. Methods to remove the placenta and to treat postoperative hemorrhage were as follows: the surgeon did not remove the placenta by hand and waited until the placenta separated from the uterine wall spontaneously; afterwards, the placenta was gently removed by hand when part of the placenta was exfoliated and another part was retained in the uterine wall. If intraoperative hemorrhage developed, gauze packing or brace sutures, such as placental bed sutures or compression sutures, alone or combined, were performed at the surgeon's discretion. Cases that did not receive any hemostatic procedures, as mentioned above, were included in our study. Hence, when the placenta did not separate, surgeons did not remove the placenta, closed the uterine wall and skin incision, and performed prophylactic uterine artery embolization (UAE). These cases were excluded from this study.

Maternal history and intraoperative information were obtained from medical charts and operative records. In all cases, US and MRI examinations for the diagnosis of placenta previa were performed by experienced obstetricians and radiologists after 30 weeks of gestation. At our institution, elective cesarean section was performed before 38 weeks of gestation according to the Guidelines for Obstetrical Practice in Japan [26]. However, if persistent antenatal bleeding over $100 \mathrm{ml}$ or uncontrollable uterine contractions occurred before the prearranged date of cesarean section, an emergency cesarean section was performed. Antenatal bleeding was defined as painless genital bleeding stemming from the placenta. The amount of intraoperative hemorrhage was measured from the time of the skin incision to the time of scar closure, based on suction count and towel weight. Massive postoperative hemorrhage 
was defined as the amount of bleeding from the end of the cesarean section procedure until $24 \mathrm{~h}$ after surgery and a blood loss over $500 \mathrm{ml}$ within 24 hours after birth [27].Lochia, which attached to maternity pads to measure postoperative hemorrhage was assessed. All included cases were categorized into two groups: Group A, massive postoperative hemorrhage and Group B, all cases without massive postoperative hemorrhage. Cases that underwent allogenic blood transfusion included patients who received blood transfusion at pre-parturition, intraoperation, and postpartum time. Placental adhesion was defined as no natural removal of the placenta after 2 minutes from the time of delivery.

We classified placenta previa into two categories according to Cali et al. [28]. If the placental edge covered the internal os, it was diagnosed as major type. If the placental edge did not cover the cervical internal os and was located in the lower uterine segment, it was classified as minor type.

Antenatal diagnosis of adherent placenta was assessed by MRI for uterine bulging, heterogeneous placenta, adjacent organ invasion, and cervical varicosities [16-18]. The pathological diagnosis of placenta accreta spectrum was performed using the placenta. We routinely used uterotonic drugs for all cases after performing cesarean section. When massive postoperative hemorrhage developed, the amount of uterotonic drugs was increased. If postoperative hemorrhage was uncontrollable, intrauterine balloon tamponade was performed. If all interventions failed, uterine artery embolization was performed.

Statistical analysis was performed using JMP Pro 14 software (SAS Institute Inc., Cary, NS, USA). Chisquared test, Fisher's exact test, and Mann-Whitney $U$ test were used to evaluate the clinical significance of clinical factors. Statistical significance was defined as a $p<0.05$.

This retrospective study was approved by the Institutional Review Board of the National Defense Medical College, Tokorozawa, Japan.

\section{Results}

During the period of this study, 242 cases with placenta previa were identified. A total of 114 cases which received hemostatic procedures during cesarean section were excluded. A total number of 128 cases were included in our study. The rate of cesarean section was $100 \%$ and incidence of massive postoperative hemorrhage was $7.8 \%$.

The clinical characteristics of both groups are presented in Table 1. All cases underwent cesarean section. There was no statistically significant difference in maternal history. According to operative information, there were more patients with suspected placental adhesion in Group A than in Group B $(p=0.006)$. Furthermore, the amount of intraoperative hemorrhage was higher in Group $A(p=0.025)$. The number of cases with antenatal diagnosis of placenta accreta spectrum was 3 in Group A and 7 in Group B. Also, final pathological findings revealed 3 cases in Group A and 2 cases in Group B with placenta accreta spectrum. 
Table 2 showed the procedure after hemorrhage. More cases in Group A received uterine artery embolization $(p=0.010)$ and allogenic blood transfusion $(p=0.003)$ as additional treatment for massive postoperative hemorrhage. In group B, 4 patients received UAE and 1 patient underwent hysterectomy due to uncontrollable massive hemorrhage.

Univariate analysis for massive postoperative hemorrhage with placenta previa revealed that placental adherence was the predictive factor (Table 3 ).

\section{Discussion}

In our study, univariate analysis revealed that only placental adhesion suspected by surgeon during cesarean section was a predictive factor for massive postoperative hemorrhage in placenta previa.

Placenta previa caused massive postoperative hemorrhage because the lower uterine segment is only weakly contractible [29]. Thus, the appropriate strategy was required.

In this study, patients who received any hemostatic procedure, such as intrauterine balloon tamponade, during cesarean section were excluded. The reasoning was that intrauterine balloon tamponade was an effective management of postoperative hemorrhage associated with placenta previa. In fact, some reports demonstrated that intrauterine balloon tamponade was effective in decreasing both intraoperative and postoperative hemorrhage $[19,23,24]$. In our institution, since March 2015, rapid insertion of intrauterine balloon tamponade has been performed to reduce hemorrhage after spontaneous separation of the placenta and has succeeded in reducing postoperative hemorrhage [25]. To design the predictive model for massive postoperative hemorrhage, we considered that the exclusion criteria were valid.

Moreover, placental adhesion was defined by the surgical findeings in this study. The definition of placental adhesion by a surgeon might be different from that of true placental adhesion using pathological examination [30]. The reason for diagnosing placental adhesion clinically rather than pathologically in our study was that massive postoperative hemorrhage of placenta previa occurred spontaneously, particularly within one day after termination [27], and timing for pathological diagnosis was impractical. Therefore, we considered the design of our study to be an appropriate clinical model to predict massive postoperative hemorrhage.

Our results suggest that any hemostatic procedures should be performed if surgeons suspect placental adhesion. Although our study did not examine the appropriate strategy for postoperative hemorrhage, the development of postoperative hemorrhage was a critical problem. According to Soyama et al., intrauterine balloon tamponade could decrease postoperative hemorrhage [25]. In addition, some studies reported the efficacy of routine prophylactic use of uterotonic drugs, such as oxytocin and tranexamic acid $[31,32]$. Further studies are required to examine the effective methods, including these, for patients with a risk factor for postoperative hemorrhage. 
This study has some limitations. First, the retrospective study focused on data from a single institution, leading to a small sample size, which did not permit the development of an appropriate strategy for this problem. Second, this study has a strong selection bias due to the exclusion criteria. Third, our study performed only univariate analysis. Further prospective studies are necessary to reveal the correlation between postoperative hemorrhage with placenta previa and risk factors and, to develop appropriate strategies.

\section{Conclusions}

Placental adhesion was the predictive factor for postoperative hemorrhage associated with placenta previa. The prevention of postoperative hemorrhage was needed for women with placental adhesion to prepare for massive postoperative hemorrhage even though a massive hemorrhage did not develop during caesarean section.

\section{Abbreviations}

US Ultrasonographic

MRI Magnetic resonance imaging

UAE Uterine artery embolization

\section{Declarations}

\section{Disclosure of potential conflicts of interest}

None.

\section{Ethics approval and consent to participate}

All procedures performed in studies involving human participants were in accordance with the ethical standards of the institutional and/or national research committee and the 1964 Declaration of Helsinki and its later amendments or comparable ethical standards. For this type of study, formal consent is not required. For the retrospective analysis, informed consent was not obtained. This study was approved by the Clinical Research Ethics Committee of the National Defense Medical College.

\section{Consent to publication}

All data were anonymized and individual consent for publication was not required.

\section{Availability of data and materials}

The datasets used and analyzed in this study are available from the corresponding author upon reasonable request. 


\section{Competing interests}

All authors declare that they have no competing interests.

\section{Funding}

The authors received no specific funding for this work.

\section{Author Contributions}

Protocol/project development: $\mathrm{HI}, \mathrm{M} \mathrm{M}$, and M T.

Data collection or management: $\mathrm{HI}, \mathrm{HS}, \mathrm{HI}, \mathrm{H} \mathrm{K}, \mathrm{K} \mathrm{T}, \mathrm{H} \mathrm{M}$, and $\mathrm{M} \mathrm{N}$.

Data analysis: $\mathrm{HI}$ and $\mathrm{M} \mathrm{M}$.

Manuscript writing/editing: $\mathrm{HI}, \mathrm{M} \mathrm{M}$, and M T.

\section{Conflict of Interest Statement and Funding/Support Statement}

None.

\section{Acknowledgements}

We would like to thank Editage (www.editage.jp) for English language editing.

\section{References}

1. Saleh Gargari S, Seify Z, Haghighi L, Khoshnood Shariati M, Mirzamoradi M. Risk factors and consequent outcomes of placenta previa: report from a referral center. Acta Med Iran 2016;54:713717.

2. Ananth CV, Smulian JC, Vintzileos AM. The effect of placenta previa on neonatal mortality: a population-based study in the United States, 1989 through 1997. Am J Obstet Gynecol 2003;88:1299-1304.

3. Walfish M, Neuman A, Wlody D. Maternal haemorrhage. Br J Anaesth 2009;103:47-56.

4. Oyelese Y, Smulian JC. Placenta previa, placenta accreta, and vasa previa. Obstet Gynecol 2006;107:927-941.

5. A Padmasekar, S Jothy. Retrospective study of massive obstetric haemorrhage and its materno fetal outcomes in a tertiary care centre. Int J Reprod Contracept Obstet Gynecol 2017;6:554-557.

6. Hasegawa J, Matsuoka R, Ichizuka K, Mimura T, Sekizawa A, Farina A, Okai T. Predisposing factors for massive hemorrhage during cesarean section in patients with placenta previa. Ultrasound Obstet Gynecol 2009;34:80-84. 
7. Baba Y, Matsubara S, Ohkuchi A, Usui R, Kuwata T, Suzuki H, Takahashi H, Suzuki M. Anterior placentation as a risk factor for massive hemorrhage during cesarean section in patients with placenta previa. J Obstet Gynecol Res 2014;40:1243-1248.

8. Jang DG, We JS, Shin JU, Choi YJ, Ko HS, Park IY, Shin JC. Maternal outcomes according to placental position in placental previa. Int J Med Sci 2011;8:439-444

9. CalõÁ G, Giambanco L, Puccio G, Forlani F. Morbidly adherent placenta: evaluation of ultrasound diagnostic criteria and differentiation of placenta accreta from percreta. Ultrasound Obstet Gynecol 2013;41:406-412.

10. Tuzovic L. Complete versus incomplete placenta previa and obstetric outcome. Int J Gynaecol Obstet 2006;93:110-117.

11. Jang DG, We JS, Shin JU, Choi YJ, Ko HS, Park IY, Shin JC. Maternal outcomes according to placental position in placental previa. Int J Med Sci 2011;8:439-444.

12. Soyama H, Miyamoto M, Ishibashi H, Takano M, Sasa H, Furuya K. Relation between birth weight and intraoperative hemorrhage during cesarean section in pregnancy with placenta previa. PLoS One 2016;11:e0167332.

13. Stafford IA, Dashe JS, Shivvers SA, Alexander JM, McIntire DD, Leveno KJ. Ultrasonographic cervical length and risk of hemorrhage in pregnancies with placenta previa. Obstet Gynecol 2010;116:595600 .

14. Ghourab S. Third-trimester transvaginal ultrasonography in placenta previa: does the shape of the lower placental edge predict clinical outcome? Ultrasound Obstet Gynecol 2001;18:103-108.

15. Zaitoun MM, El Behery MM, Abd El Hameed AA, Soliman BS. Does cervical length and the lower placental edge thickness measurement correlates with clinical outcome in cases of complete placenta previa? Arch Gynecol Obstet 2011;284:867-873.

16. Baughman WC, Corteville JE, Shah RR. Placenta accreta: spectrum of US and MR imaging findings. Radiographics 2008;28:1905-1916.

17. D’Antonio F, lacovella C, Palacios-Jaraquemada J, Bruno CH, Manzoli L, Bhide A. Prenatal identification of invasive placentation using magnetic resonance imaging: systematic review and meta-analysis. Ultrasound Obstet Gynecol 2014;44:8-16.

18. Ishibashi H, Miyamoto M, Shinnmoto H, Murakami W, Soyama H, Nakatsuka M, Natsuyama T, Yoshida M, Takano M, Furuya K. Cervical varicosities may predict placenta accreta in posterior placenta previa: a magnetic resonance imaging study. Arch Gynecol Obstet 2017;296:731-736.

19. Bakri YN, Amri A, Abdul Jabbar F. Tamponade-balloon for obstetrical bleeding. Int J Gynaecol Obstet 2001;74:139-142.

20. Carnevale FC, Kondo MM, de Oliveira Sousa W Jr, Santos AB, da Motta Leal Filho JM, Moreira AM, et al. Perioperative temporary occlusion of the internal iliac arteries as prophylaxis in cesarean section at risk of hemorrhage in placenta accreta. Cardiovasc Intervent Radiol 2011;34:758-764.

21. Lee HJ, Lee YJ, Ahn EH, Kim HC, Jung SH, Chang SW, et al. Risk factors for massive postpartum bleeding in pregnancies in which incomplete placenta previa are located on the posterior uterine wall. 
Obstet Gynecol Sci 2017;60:520-526.

22. Lal AK, Hibbard JU. Placenta previa: an outcome-based cohort study in a contemporary obstetric population. Arch Gynecol Obstet 2015;292:299-305.

23. Alouini S, Bedouet L, Ramos A, Ceccaldi C, Evrard ML, Khadre K. Bakri balloon tamponade for severe post-partum haemorrhage: efficiency and fertility outcomes. J Gynecol Obstet Biol Reprod (Paris) 2015;44:171-175.

24. Maher MA, Abdelaziz A. Comparison between two management protocols for postpartum hemorrhage during cesarean section in placenta previa: Balloon protocol versus non-balloon protocol. J Obstet Gynaecol Res 2017;43:447-455.

25. Soyama H, Miyamoto M, Sasa H, Ishibashi H, Yoshida M, Nakatsuka M, et al. Effect of routine rapid insertion of Bakri balloon tamponade on reducing hemorrhage from placenta previa during and after cesarean section. Arch Gynecol Obstet. 2017;296:469-474.

26. Minakami H, Maeda T, Fujii T, Hamada H, litsuka Y, Itakura A, et al. Guidelines for obstetrical practice in Japan: Japan society of obstetrics and gynecology (JSOG) and Japan association of obstetricians and gynecologists (JAOG) 2014 edition. J Obstet Gynaecol Res 2014;40:1469e99.

27. World Health Organization. WHO recommendations for the prevention and treatment of postpartum haemorrhage. Geneva: World Health Organization; 2012.3.

https://apps.who.int/iris/bitstream/handle/10665/75411/9789241548502_eng.pdf?sequence=1 accessed 18 April 2019.

28. Calì G, Giambanco L, Puccio G, Forlani F. Morbidly adherent placenta: evaluation of ultrasound diagnostic criteria and differentiation of placenta accreta from percreta. Ultrasound Obstet Gynecol 2013;41:406-412.

29. Oyelese $Y$, Ananth CV. Postpartum hemorrhage: epidemiology, risk factors, and causes. Clin Obstet Gynecol 2010;53:147-156.

30. Eller AG, Porter TF, Soisson P, Silver RM. Optimal management strategies for placenta accreta. BJOG 2009;116:648-654.

31. Westhoff G, Cotter AM, Tolosa JE. Prophylactic oxytocin for the third stage of labour to prevent postpartum haemorrhage. Cochrane Database Syst Rev 2013;10:CD001808.

32. Lakshmi SD, Abraham R. Role of prophylactic tranexamic acid in reducing blood loss during elective caesarean section: a randomized controlled study. J Clin Diagn Res 2016;10:QC17-QC21.

\section{Tables}

Table 1: Characteristics of patients with placenta previa. 


\begin{tabular}{|c|c|c|c|}
\hline Clinical factors & $\begin{array}{c}\text { Group A } \dagger \\
n=10\end{array}$ & $\begin{array}{c}\text { Group B } \neq \\
n=118\end{array}$ & $p$-value \\
\hline \multicolumn{4}{|l|}{ Maternal history } \\
\hline Maternal age $(\mathrm{y})$, mean \pm SD $\S$ & $33.9 \pm 3.6$ & $33.5 \pm 4.9$ & 0.814 \\
\hline Gestational age $(w)$, mean \pm SD & $36.4 \pm 1.5$ & $36.4 \pm 1.5$ & 0.859 \\
\hline Primipara, number (\%) & $3(30.0)$ & $61(51.69)$ & 0.324 \\
\hline ART 9 pregnancy, number (\%) & $2(20.0)$ & $8(6.78)$ & 0.176 \\
\hline Repeat cesarean section, number (\%) & $2(20.0)$ & $15(12.7)$ & 0.621 \\
\hline Tocolytic agent use, number (\%) & $3(30.0)$ & $44(37.3)$ & 0.745 \\
\hline Complication of myoma, number (\%) & $0(0.0)$ & $12(10.2)$ & 0.597 \\
\hline Placental classification & & & 0.192 \\
\hline Major placenta, number (\%) & $7(70.0)$ & $54(45.8)$ & \\
\hline Minor placenta, number (\%) & $3(30.0)$ & $64(54.2)$ & \\
\hline Placenta on anterior wall, number (\%) & $2(20.0)$ & $17(14.41)$ & 0.643 \\
\hline Antenatal bleeding, number (\%) & $5(50.0)$ & $36(30.51)$ & 0.289 \\
\hline \multicolumn{4}{|l|}{ Operative information } \\
\hline Emergency delivery, number (\%) & $3(30.0)$ & $30(25.4)$ & 0.717 \\
\hline Baby weight (g), mean \pm SD & $2660.0 \pm 387.6$ & $2549.6 \pm 421.2$ & 0.667 \\
\hline Placental adhesion, number (\%) & $3(30.0)$ & $3(2.5)$ & 0.006 \\
\hline Operation time (min), mean \pm SD & $80.9 \pm 61.0$ & $55.3 \pm 24.3$ & 0.267 \\
\hline Intraoperative hemorrhage $(\mathrm{ml})$, mean \pm SD & $1713.1 \pm 898.6$ & $1120.1 \pm 531.5$ & 0.025 \\
\hline Postoperative hemorrhage $(\mathrm{ml})$, mean \pm SD & $1438.8 \pm 920.2$ & $113.4 \pm 89.4$ & $<0.001$ \\
\hline
\end{tabular}

†Group A; patients with massive postoperative hemorrhage.

‡Group B; patients without massive postoperative hemorrhage.

- SD; standard deviation,

- 9ART; assisted reproductive technologies

Table 2: Comparison of additional treatment for postoperative hemorrhage.

\begin{tabular}{lccc}
\hline Additional treatment for postoperative hemorrhage & Group A $\dagger$ & Group B & $p$-value \\
& $\mathrm{n}=10$ & $\mathrm{n}=118$ & \\
\hline Uterotonic drugs§, number (\%) & $10(100.0)$ & $118(100.0)$ & 0.999 \\
\hline Intrauterine balloon tamponade, number (\%) & $1(10.0)$ & $1(0.9)$ & 0.151 \\
\hline Uterine artery embolization, number (\%) & $3(30.0)$ & $4(3.39)$ & 0.010 \\
\hline Allogenic blood transfusion, number (\%) & $5(50.0)$ & $11(9.3)$ & 0.003 \\
\hline Total abdominal hysterectomy, number (\%) & $1(10.0)$ & $1(0.9)$ & 0.151 \\
\hline
\end{tabular}

tGroup A; patients with massive postoperative hemorrhage. 
‡Group B; patients without massive postoperative hemorrhage.

- Uterotonic drugs; several drugs including oxytocin, methylergometrine, and dinoprost.

Table 3: Univariate analysis for postpartum hemorrhage in placenta previa's cases.

\begin{tabular}{|c|c|c|c|c|}
\hline Clinical factors & & $\begin{array}{l}\text { Odds } \\
\text { Ratio }\end{array}$ & $(95 \% \mathrm{CI}+\square$ & $\begin{array}{c}p- \\
\text { value }\end{array}$ \\
\hline Age & $\geqq 35$ vs $<35$ & 0.818 & $\begin{array}{c}(0.219- \\
3.049)\end{array}$ & 0.764 \\
\hline $\begin{array}{l}\text { Repeat cesarean } \\
\text { section }\end{array}$ & positive vs negative & 1.717 & $\begin{array}{l}(0.333- \\
8.862)\end{array}$ & 0.519 \\
\hline Placental classification & major vs minor & 2.765 & $\begin{array}{l}(0.682- \\
11.216)\end{array}$ & 0.155 \\
\hline Placental location & anterior vs posterior & 1.485 & $\begin{array}{l}(0.290- \\
7.599)\end{array}$ & 0.635 \\
\hline Antenatal bleeding & positive vs negative & 2.278 & $\begin{array}{l}(0.621- \\
8.358)\end{array}$ & 0.215 \\
\hline Placental adhesion & positive vs negative & 16.429 & $\begin{array}{l}(2.790- \\
96.729)\end{array}$ & 0.002 \\
\hline $\begin{array}{l}\text { Intraoperative } \\
\text { hemorrhage }\end{array}$ & $\mathrm{ml}$ & 2.933 & $\begin{array}{l}(0.794- \\
10.839)\end{array}$ & 0.107 \\
\hline
\end{tabular}

†CI; confidence interval 